\title{
RECURRENCE RELATIONS FOR POLYNOMIALS OBTAINED BY ARITHMETIC FUNCTIONS
}

\author{
BERNHARD HEIM, FLORIAN LUCA, AND MARKUS NEUHAUSER
}

\begin{abstract}
Families of polynomials associated to arithmetic functions $g(n)$ are studied. The case $g(n)=\sigma(n)$, the divisor sum, dictates the non-vanishing of the Fourier coefficients of powers of the Dedekind eta function. The polynomials $P_{n}^{g}(X)$ are defined by $n$-term recurrence relations. For the case that $g(x)$ is a polynomial of degree $d$, we prove that at most a $d+2$ term recurrence relation is needed. For the special case $g(x)=x$ we obtain explicit formulas and results.
\end{abstract}

\section{INTRODUCTION}

Let $q=e^{2 \pi i \tau}$ and $\tau$ be in the upper complex half-plane. We consider

$$
\exp \left(X \sum_{n \geq 1} g(n) \frac{q^{n}}{n}\right)=\sum_{n \geq 0} P_{n}^{g}(X) q^{n},
$$

where $g: \mathbb{N} \rightarrow \mathbb{N}$ is an arithmetic function normalized such that $g(1)=1$. This implies a recursive definition of the polynomials $P_{n}^{g}(X)$ :

$$
P_{n}^{g}(X)=\frac{X}{n} \sum_{k=1}^{n} g(k) P_{n-k}^{g}(X) \quad \text { for all } \quad n \geq 1,
$$

where $P_{0}^{g}(X):=1$.

Let for the moment $g(n)=\sigma(n)=\sum_{t \mid n} t$ be the sum of all divisors of $n$. Then we obtain the powers of the Euler product

$$
\prod_{n \geq 1}\left(1-q^{n}\right)^{-X}=\exp \left(X \sum_{n \geq 1} \sigma(n) \frac{q^{n}}{n}\right) .
$$

This is essentially an identity involving powers of the Dedekind eta function $\eta(\tau)=q^{1 / 24} \prod_{n \geq 1}\left(1-q^{n}\right)$ (see [On03] for more details). Hence, the roots of $P_{n}^{\sigma}(X)$ dictate the vanishing properties of the $n$-th Fourier coefficients of powers of the Dedekind eta function (see [HNR17, HLN18, HNR18] for more details and the connection to the Lehmer conjecture [Le47]).

2010 Mathematics Subject Classification. Primary 05A17, 11C08; Secondary 11B83, 11F20, 11F30.

Key words and phrases. Arithmetic functions, Dedekind eta function, polynomials, recurrence relation. 
Explicit formulas for $P_{n}^{g}(X)$ in the case $g(n)=1$ for all $n \in \mathbb{N}$ are given by

$$
\exp \left(X \sum_{n \geq 1} \frac{q^{n}}{n}\right)=(1-q)^{-X}=\sum_{n \geq 0}\left(\begin{array}{c}
-X \\
n
\end{array}\right)(-q)^{n}
$$

Hence,

$$
P_{n}^{g}(X)=\frac{1}{n !} X(X+1) \cdots(X+n-1)=\frac{1}{n !} \sum_{k=1}^{n} S_{n, k} X^{k}
$$

for $n \geq 1$ and $P_{0}^{g}(X)=1$. Here, the coefficients $S_{n, k}$ are the Stirling numbers of the first kind.

The aim of this paper is to study the polynomials $P_{n}^{g}(X)$ for some interesting functions $g(n)$ particularly for $g(n)=n$. In this case the coefficients of $X^{k}$ of the polynomials $n ! P_{n}^{g}(X)$ are the so-called Lah numbers $\frac{n !}{k !}\left(\begin{array}{c}n-1 \\ k-1\end{array}\right)$ ([Ai07]) and the polynomials are related to the generalized Laguerre polynomials [Do16]

$$
L_{n}^{(\alpha)}(X)=\sum_{k=0}^{n}\left(\begin{array}{c}
n+\alpha \\
n-k
\end{array}\right) \frac{(-X)^{k}}{k !} \quad(\alpha>-1) .
$$

In the above case, we show that the sequence of the polynomials $P_{n}^{g}(X)$ satisfies a three term recurrence relation. More generally, we show that if $g(x)$ is a polynomial of degree $d$, then $\left(P_{n}^{g}(X)\right)_{n \geq 0}$ satisfies a recurrence relation of order at most $d+2$. From the properties of orthogonal polynomials [Do16], we can also derive that in the case $g(x)=x$ the roots are all simple and real. Putting $\widetilde{P}_{n}^{g}(X)=X^{-1} P_{n}^{g}(X)$, we show that $\widetilde{P}_{n}^{g}(X)$ is irreducible in this case.

\section{Recurrence Relation for the CASE When $g(x)$ is a polynomial}

In this section, we prove the following.

Theorem 1. Let $g(x)$ be a polynomial of degree $d$. For $0 \leq m, j \leq d$ define

$$
\begin{aligned}
& b_{m}=\sum_{\mu=0}^{m}(-1)^{\mu}\left(\begin{array}{l}
m \\
\mu
\end{array}\right) g(-\mu), \\
& c_{j}=(-1)^{j}\left(b_{0}\left(\begin{array}{l}
d \\
j
\end{array}\right)+\sum_{\iota=1}^{d-j} \iota b_{\iota}\left(\begin{array}{c}
d-\iota \\
j
\end{array}\right)\right) .
\end{aligned}
$$

Then the polynomials $P_{n}^{g}(X)$ satisfy the following $d+2$ term recurrence relations with $P_{0}^{g}(X)=1$ and

$$
P_{n+1}^{g}(X)=\frac{1}{n+1} \sum_{j=0}^{\min \{n, d\}}\left(c_{j} X+(-1)^{j}\left(\begin{array}{l}
d+1 \\
j+1
\end{array}\right)(n-j)\right) P_{n-j}^{g}(X) .
$$


For the proof, we want to express our polynomial $g(x)$ in terms of the basis

$$
\{1, x, x(x+1), x(x+1)(x+2) / 2 !, \ldots, x(x+1)(x+2) \cdots(x+d) / d !, \ldots\} .
$$

Hence, we first show how to determine the coefficients in terms of this basis. These are exactly the $b_{m}$ as stated in the theorem.

Lemma 1. Let $Q(x)=\sum_{m=0}^{d} b_{m} \frac{x(x+1) \cdots(x+m-1)}{m !}$. Then the coefficients $b_{m}$ for $0 \leq m \leq d$ can be recovered as $b_{m}=\sum_{\mu=0}^{m}(-1)^{\mu}\left(\begin{array}{c}m \\ \mu\end{array}\right) Q(-\mu)$.

We give a proof of this lemma at the end of this section.

Proof of Theorem 1. We have,

$$
\frac{g(n)}{n}=\frac{b_{0}}{n}+\sum_{m=1}^{d} b_{m} \frac{(n+1) \cdots(n+m-1)}{(m-1) !} .
$$

Then

$$
\begin{aligned}
X \sum_{n \geq 1} g(n) \frac{q^{n}}{n} & =X\left(\sum_{n \geq 1} \frac{b_{0}}{n}+\sum_{m=1}^{d} b_{m} \frac{(n+1) \cdots(n+m-1)}{(m-1) !}\right) q^{n} \\
& =X b_{0} \sum_{n \geq 1} \frac{q^{n}}{n}+X \sum_{m=1}^{d} b_{m} \sum_{n \geq 1} \frac{(n+1) \cdots(n+m-1)}{(m-1) !} q^{n}
\end{aligned}
$$

for $|q|<1$. The first inner sum is $-\log (1-q)$. The generic inner sum for $m \geq 1$ is

$$
\begin{aligned}
\sum_{n \geq 1} \frac{(n+1) \cdots(n+m-1)}{(m-1) !} q^{n} & =\frac{1}{(m-1) !}\left(\frac{\partial^{m-1}}{\partial q^{m-1}}\left(\frac{1}{1-q}\right)\right)-1 \\
& =\frac{1}{(1-q)^{m}}-1 .
\end{aligned}
$$

Our exponential generating function is therefore

$$
\begin{aligned}
F(q, X) & :=\exp \left(X \sum_{n \geq 1} g(n) \frac{q^{n}}{n}\right) \\
& =\exp \left(-b_{0} X \log (1-q)+X \sum_{m=1}^{d} b_{m}\left(\frac{1}{(1-q)^{m}}-1\right)\right) .
\end{aligned}
$$


Thus,

$$
\begin{aligned}
& \frac{\partial}{\partial q} F(q, X) \\
= & F(q, X) \frac{\partial}{\partial q}\left(-b_{0} X \log (1-q)+X \sum_{m=1}^{d} b_{m}\left(\frac{1}{(1-q)^{m}}-1\right)\right) \\
= & X F(q, X)\left(\frac{b_{0}}{1-q}+\sum_{m=1}^{d} \frac{m b_{m}}{(1-q)^{m+1}}\right) .
\end{aligned}
$$

Thus,

$$
(1-q)^{d+1} \frac{\partial}{\partial q} F(q, X)=X F(q, X)\left(b_{0}(1-q)^{d}+\sum_{m=1}^{d} m b_{m}(1-q)^{d-m}\right),
$$

or

$$
\begin{aligned}
& \left(\sum_{j=0}^{d+1}(-1)^{j}\left(\begin{array}{c}
d+1 \\
j
\end{array}\right) q^{j}\right) \times\left(\sum_{n \geq 1} n P_{n}^{g}(X) q^{n-1}\right) \\
= & X\left(\sum_{n \geq 0} P_{n}^{g}(X) q^{n}\right)\left(b_{0}(1-q)^{d}+\sum_{m=1}^{d} m b_{m}(1-q)^{d-m}\right) .
\end{aligned}
$$

Now

$$
b_{0}(1-q)^{d}+\sum_{m=1}^{d} m b_{m}(1-q)^{d-m}=\sum_{j=0}^{d} c_{j} q^{j}
$$

where

$$
c_{j}=(-1)^{j} b_{0}\left(\begin{array}{l}
d \\
j
\end{array}\right)+\sum_{m=1}^{d-j} m b_{m}(-1)^{j}\left(\begin{array}{c}
d-m \\
j
\end{array}\right) .
$$

Thus,

$$
\begin{aligned}
& \left(\sum_{j=0}^{d+1}(-1)^{j}\left(\begin{array}{c}
d+1 \\
j
\end{array}\right) q^{j}\right) \times\left(\sum_{n \geq 1} n P_{n}^{g}(X) q^{n-1}\right) \\
= & X\left(\sum_{n \geq 0} P_{n}^{g}(X) q^{n}\right)\left(\sum_{j=0}^{d} c_{j} q^{j}\right) .
\end{aligned}
$$

Expanding and identifying the coefficients of $q^{n}$ on left and right-hand sides above we get

$$
\sum_{j=0}^{\min \{d, n\}+1}(-1)^{j}\left(\begin{array}{c}
d+1 \\
j
\end{array}\right)(n+1-j) P_{n+1-j}^{g}(X)=X \sum_{j=0}^{\min \{d, n\}} c_{j} P_{n-j}^{g}(X) .
$$


Hence, we obtain

$$
P_{n+1}^{g}(X)=\frac{1}{n+1} \sum_{j=0}^{\min \{n, d\}}\left(c_{j} X+(-1)^{j}\left(\begin{array}{l}
d+1 \\
j+1
\end{array}\right)(n-j)\right) P_{n-j}^{g}(X) .
$$

Example 1. We give an explicit two term recurrence relation for $\left(P_{n}^{g}(X)\right)_{n \geq 0}$ when $g(n)=1$.

For $g(n)=1$ we have in the preceding notation $d=0, b_{0}=1$, and $c_{0}=1$. This leads to $P_{0}^{g}(X)=1$ and

$$
P_{n+1}^{g}(X)=\frac{1}{n+1}(X+n) P_{n}^{g}(X)
$$

for all $n \geq 0$, which yields exactly the polynomials (2) from the Introduction.

Example 2. We give an explicit three term recurrence relation for $\left(P_{n}^{g}(X)\right)_{n \geq 0}$ when $g(n)=n$.

For $g(n)=n$ we have in the preceding notation $d=1, b_{0}=0, b_{1}=1$, $c_{0}=1$, and $c_{1}=0$. This leads to $P_{0}^{g}(X)=1, P_{1}^{g}(X)=X P_{0}^{g}(X)$, and

$$
P_{n+1}^{g}(X)=\frac{1}{n+1}\left((2 n+X) P_{n}^{g}(X)-(n-1) P_{n-1}^{g}(X)\right)
$$

for all $n \geq 1$.

Proof of Lemma 1. We obtain for $x=-N$ and $0 \leq N \leq d$ :

$$
\begin{aligned}
& \sum_{m=0}^{d} \sum_{\mu=0}^{m}(-1)^{\mu}\left(\begin{array}{l}
m \\
\mu
\end{array}\right) Q(-\mu) \frac{-N(-N+1) \cdots(-N+m-1)}{m !} \\
= & \sum_{m=0}^{N} \sum_{\mu=0}^{m}(-1)^{\mu}\left(\begin{array}{l}
m \\
\mu
\end{array}\right) Q(-\mu)(-1)^{m}\left(\begin{array}{l}
N \\
m
\end{array}\right) \\
= & \sum_{\mu=0}^{N} \sum_{m=\mu}^{N}(-1)^{m+\mu} \frac{N !}{\mu !(m-\mu) !(N-m) !} Q(-\mu) \\
= & \sum_{\mu=0}^{N} \sum_{m=0}^{N-\mu}(-1)^{m} \frac{N !}{\mu ! m !(N-m-\mu)} Q(-\mu) \\
= & \sum_{\mu=0}^{N} \sum_{m=0}^{N-\mu}(-1)^{m}\left(\begin{array}{c}
N-\mu \\
m
\end{array}\right)\left(\begin{array}{l}
N \\
\mu
\end{array}\right) Q(-\mu)=\sum_{\mu=0}^{N} \delta_{N, \mu}\left(\begin{array}{c}
N \\
\mu
\end{array}\right) Q(-\mu) \\
= & Q(-N) .
\end{aligned}
$$




\section{IrReducibility of $\widetilde{P}_{n}^{g}(X)$ When $g(n)=n$}

In this case $P_{n}(X):=P_{n}^{g}(X)$ has the following closed form (compare with [HN18]):

(4) $P_{n}(X)=\frac{1}{n !} \sum_{k=1}^{n} \frac{n !}{k !}\left(\begin{array}{c}n-1 \\ k-1\end{array}\right) X^{k}=X \sum_{k=0}^{n-1}\left(\begin{array}{c}n-1 \\ k\end{array}\right) \frac{X^{k}}{(k+1) !}=X \widetilde{P}_{n}(X)$.

The coefficients of the polynomial $n ! P_{n}(X)$ are the so-called Lah numbers $\frac{n !}{k !}\left(\begin{array}{c}n-1 \\ k-1\end{array}\right)$ (compare with e. g. [Ai07]).

Theorem 2. $\widetilde{P}_{n}(X)$ is irreducible for all $n \geq 1$.

Theorem 3. For $g(n)=n$ all the roots of the polynomials $P_{n}(X)=P_{n}^{g}(X)$ are simple and non-positive real numbers. Additionally the negative roots are interlacing from $n$ to $n+1$.

By interlacing we mean that if $s_{n, 1}<\ldots<s_{n, n-1}<s_{n, n}=0$ are the roots of $P_{n}(X)$ then $s_{n+1,1}<s_{n, 1}<s_{n+1,2}<s_{n, 2}<\ldots<s_{n+1, n-1}<$ $s_{n, n-1}<s_{n+1, n}<0$.

For the proofs of the preceding two theorems the important thing to notice is the following lemma.

Lemma 2. For $n \geq 1$

$$
P_{n}(X)=\frac{X}{n} L_{n-1}^{(1)}(-X)
$$

where $L_{n-1}^{(1)}(X)=\sum_{k=0}^{n-1}\left(\begin{array}{l}(n-1)+1 \\ (n-1)-k\end{array}\right) \frac{(-X)^{k}}{k !}$ is the $n-1$ st generalized Laguerre polynomial with $\alpha=1$ (compare with (3) form the Introduction).

Proof. From the explicit formula (4) we obtain

$$
\begin{aligned}
P_{n}(X) & =X \sum_{k=0}^{n-1}\left(\begin{array}{c}
n-1 \\
k
\end{array}\right) \frac{X^{k}}{(k+1) !}=X \sum_{k=0}^{n-1} \frac{(n-1) !}{k !(n-1-k) !} \frac{X^{k}}{(k+1) !} \\
& =\frac{X}{n} \sum_{k=0}^{n-1} \frac{n !}{(k+1) !(n-1-k) !} \frac{X^{k}}{k !}=\frac{X}{n} \sum_{k=0}^{n-1}\left(\begin{array}{c}
n \\
n-1-k
\end{array}\right) \frac{X^{k}}{k !} \\
& =\frac{X}{n} L_{n-1}^{(1)}(-X) .
\end{aligned}
$$

Theorem 2 now follows from the irreducibility of $L_{n-1}^{(1)}(X)$ shown by Schur in [Sc31].

Theorem 3 follows from the respective properties of the family of orthogonal polynomials $L_{n-1}^{(1)}(X)$ (see e. g. [Do16]).

Remark 1. Other proofs of Theorem 3 are also possible: We could show directly that the polynomials $\widetilde{P}_{n}(X)$ form a system of orthogonal polynomials with respect to the measure on $(-\infty, 0]$ with Lebesgue density $x \mapsto(-x) e^{x}$. 
Or we could use the recurrence relation from Example 2 from Section 2 and apply [WY05, Theorem 1].

\section{Partial Results When $g(n)=n^{2}$}

In this case $P_{n}(X):=P_{n}^{g}(X)$ has the following closed form (compare [HN18]):

$$
P_{n}(X)=X \sum_{k=0}^{n-1} \frac{1}{(k+1) !}\left(\begin{array}{c}
n+k \\
2 k+1
\end{array}\right) X^{k}=X \widetilde{P}_{n}(X) .
$$

Note that

$$
\widetilde{P}_{n}(X)=\sum_{k=0}^{n-1} A_{n, k} \frac{X^{k}}{(k+1) !}, \quad A_{n, k} \in \mathbb{Z} .
$$

In our case $A_{n, k}=\left(\begin{array}{c}n+k \\ 2 k+1\end{array}\right)$.

Remark 2. There is a theorem of Schur [Sc29, Satz IV] and a generalization of Allen and Filaseta [AF03, Theorem 2] concerning the irreducibility of polynomials of the form (6). But both require $\left|A_{n, 0}\right|=1$ whereas in our case (5) we have $A_{n, 0}=n$. Schur used [Sc29, Satz IV] to prove the irreducibility of the generalized Laguerre polynomials $L_{n-1}^{(1)}(X)$ in [Sc31].

Proposition 1. If $n-1$ is prime then $\widetilde{P}_{n}(X)$ with $A_{n, k}=\left(\begin{array}{c}n+k \\ 2 k+1\end{array}\right), k=$ $0, \ldots, n-1$, as in (5) is irreducible.

Proof. If we multiply with $n$ ! the polynomial $n ! \widetilde{P}_{n}(X)$ has the integer coefficients $\frac{n !}{(k+1) !} A_{n, k}$. Hence, $n-1 \mid \frac{n !}{(k+1) !} A_{n, k}$ for $k=0, \ldots, n-3$ and $n-1 \mid \frac{n !}{(n-1) !} A_{n, n-2}=(2 n-2) n$. If $n-1$ is prime then $(n-1)^{2}$ does not divide $n ! n=\frac{n !}{1 !} A_{n, 0}$. So, the criterion of Eisenstein yields in this case that $\widetilde{P}_{n}(X)$ is irreducible.

Remark 3 . Note that $n-1$ prime is the only case where Eisenstein's criterion applies to the polynomials (5) since we need a prime $p$ that divides in particular $\frac{n !}{(n-1) !} A_{n, n-2}=2(n-1) n$. In case $p \mid n$, obviously $p^{2} \mid n ! n=n ! A_{n, 0}$. In case $p \mid n-1$ but $p \neq n-1$, then also $p^{2} \mid n^{2}(n-1) \frac{(n-2) !}{p !} p !=n ! A_{n, 0}$.

We used a computer to check that the above polynomials are irreducible for all $n \leq 100$.

Proposition 2. The polynomials $P_{n}(X)$ satisfy a four term recurrence relation with

$P_{0}(X)=1, P_{1}(X)=X P_{0}(X), P_{2}(X)=\frac{1}{2}\left((X+3) P_{1}(X)+X P_{0}(X)\right)$,

and

$$
\begin{aligned}
& P_{n+1}(X)= \\
& \frac{1}{n+1}\left(3(X+n) P_{n}(X)+(X-3 n+3) P_{n-1}(X)+(n-2) P_{n-2}(X)\right),
\end{aligned}
$$


for $n \geq 2$.

Proof. In the notation of Theorem 1 for $g(n)=n^{2}$ we have $d=2, b_{0}=0$, $b_{1}=-1, b_{2}=2, c_{0}=3, c_{1}=1$, and $c_{2}=0$. Using Theorem 1 this leads to the stated four term recurrence relation.

\section{A NECESSARY CONDITION FOR A THREE TERM RECURRENCE RELATION FORMULA IN THE CASE OF ARBITRARY $g(n)$}

We are going to prove a necessary condition that the $P_{n}^{g}(X)$ are a family of orthogonal polynomials and hence fulfill a three term recurrence relation of the form

$$
X P_{n}^{g}(X)=\alpha_{n} P_{n+1}^{g}(X)+\beta_{n} P_{n}^{g}(X)+\gamma_{n} P_{n-1}^{g}(X)
$$

for some $\alpha_{n}, \beta_{n}$, and $\gamma_{n}$. From the proof of the criterion the quite remarkable fact can be observed that it does not depend on $n$ in the sense that if the criterion is not fulfilled also (7) cannot be fulfilled for any $n \geq 2$.

Proposition 3. Let $g(n)$ be an arbitrary arithmetic function with $g(1)=1$. A necessary condition that the $P_{n}^{g}(X)$ are a family of orthogonal polynomials is $g(2)^{3}-2 g(2) g(3)+g(4)=0$.

Corollary 1. For $g(n)=n^{\ell}, \ell \neq 0,1$, the polynomials $P_{n}^{g}(X)$ do not satisfy a three term recurrence relation of the form (7) and hence they are not a system of orthogonal polynomials.

Remark 4. This allows us to complement the result of Proposition 2 for $g(n)=n^{2}$ since $2=\ell \neq 0,1$.

Of course we also could just have checked directly that $g(2)^{3}-2 g(2) g(3)+$ $g(4)=64-72+16=8 \neq 0$.

Proof of Corollary 1. For $g(x)=x^{\ell}, x>0$, we obtain $g^{\prime \prime}(x)=(\ell-1) \ell x^{\ell-2}$ for the second derivative which is positive for $\ell>1$ or $\ell<0$ and negative for $0<\ell<1$. This implies $g(x)$ is strictly concave up for $\ell>1$ or $\ell<0$ and strictly concave down for $0<\ell<1$. Hence $3^{\ell}<\left(2^{\ell}+4^{\ell}\right) / 2$ in case $\ell>1$ or $\ell<0$ and $3^{\ell}>\left(2^{\ell}+4^{\ell}\right) / 2$ in case $0<\ell<1$. However we only need that we do not have equality in these cases. Hence $g(2)^{3}-2 g(2) g(3)+g(4)=$ $2^{3 \ell}-2^{\ell+1} 3^{\ell}+4^{\ell}=2^{\ell}\left(4^{\ell}-2 \cdot 3^{\ell}+2^{\ell}\right) \neq 0$ for $\ell \neq 0,1$.

In the proof of Proposition 3 we need explicitly the four highest coefficients of $P_{n}^{g}(X)$. This will be done separately in the following. 
Lemma 3. Let $g(n)$ be an arbitrary arithmetic function with $g(1)=1$. Let $P_{n}^{g}(X)=\sum_{k=0}^{n} A_{n, k} X^{k}$. Then

$$
\begin{aligned}
A_{n, n} & =\frac{1}{n !}, \quad n \geq 0, \\
A_{n, n-1} & =\frac{1}{n !} g(2)\left(\begin{array}{l}
n \\
2
\end{array}\right), \quad n \geq 1, \\
A_{n, n-2} & =\frac{1}{n !}\left(3 g(2)^{2}\left(\begin{array}{l}
n \\
4
\end{array}\right)+2 g(3)\left(\begin{array}{l}
n \\
3
\end{array}\right)\right), \quad n \geq 2, \\
A_{n, n-3} & =\frac{1}{n !}\left(15 g(2)^{3}\left(\begin{array}{l}
n \\
6
\end{array}\right)+20 g(2) g(3)\left(\begin{array}{l}
n \\
5
\end{array}\right)+6 g(4)\left(\begin{array}{l}
n \\
4
\end{array}\right)\right), \quad n \geq 3 .
\end{aligned}
$$

where a binomial coefficient $\left(\begin{array}{l}n \\ k\end{array}\right)$ is assumed to be 0 in case $n<k$.

We give a proof at the end of this section and first turn to the proof of Proposition 3.

Proof of Proposition 3. We have

$$
(n+1) ! P_{n+1}^{g}(X)-n ! X P_{n}^{g}(X)=\sum_{k=1}^{n+1}\left((n+1) ! A_{n+1, k}-n ! A_{n, k-1}\right) X^{k} .
$$

With the previous lemma for $k=n+1$ in particular, we get that

$$
(n+1) ! A_{n+1, n+1}-n ! A_{n, n}=0 .
$$

For $k=n, n-1, n-2$ we obtain

$$
\text { (8) } \begin{aligned}
&(n+1) ! A_{n+1, n}-n ! A_{n, n-1}=g(2)\left(\begin{array}{c}
n+1 \\
2
\end{array}\right)-g(2)\left(\begin{array}{l}
n \\
2
\end{array}\right)=g(2) n, \\
&(n+1) ! A_{n+1, n-1}-n ! A_{n, n-2} \\
&= 3 g(2)^{2}\left(\begin{array}{c}
n+1 \\
4
\end{array}\right)+2 g(3)\left(\begin{array}{c}
n+1 \\
3
\end{array}\right)-3 g(2)^{2}\left(\begin{array}{l}
n \\
4
\end{array}\right)-2 g(3)\left(\begin{array}{l}
n \\
3
\end{array}\right) \\
&= 3 g(2)^{2}\left(\begin{array}{l}
n \\
3
\end{array}\right)+2 g(3)\left(\begin{array}{c}
n \\
2
\end{array}\right), \\
&(n+1) ! A_{n+1, n-2}-n ! A_{n, n-3} \\
&= 15 g(2)^{3}\left(\begin{array}{c}
n+1 \\
6
\end{array}\right)+20 g(2) g(3)\left(\begin{array}{c}
n+1 \\
5
\end{array}\right)+6 g(4)\left(\begin{array}{c}
n+1 \\
4
\end{array}\right) \\
&-15 g(2)^{3}\left(\begin{array}{l}
n \\
6
\end{array}\right)-20 g(2) g(3)\left(\begin{array}{c}
n \\
5
\end{array}\right)-6 g(4)\left(\begin{array}{l}
n \\
4
\end{array}\right) \\
&= 15 g(2)^{3}\left(\begin{array}{c}
n \\
5
\end{array}\right)+20 g(2) g(3)\left(\begin{array}{l}
n \\
4
\end{array}\right)+6 g(4)\left(\begin{array}{l}
n \\
3
\end{array}\right) .
\end{aligned}
$$

From (8) we observe that the coefficient of $X^{n}$ of

$$
(n+1) ! P_{n+1}^{g}(X)-n ! X P_{n}^{g}(X)-n ! g(2) n P_{n}^{g}(X)
$$

equals 0 . Since the degree of $P_{n}^{g}(X)$ is $n$ the coefficient of $X^{n+1}$ remains 0 . 
For the coefficients of $X^{n-1}$ and $X^{n-2}$ we obtain

$$
\begin{aligned}
& (n+1) ! A_{n+1, n-1}-n ! A_{n, n-2}-n ! g(2) n A_{n, n-1} \\
= & 3 g(2)^{2}\left(\begin{array}{l}
n \\
3
\end{array}\right)+2 g(3)\left(\begin{array}{l}
n \\
2
\end{array}\right)-g(2)^{2} n\left(\begin{array}{l}
n \\
2
\end{array}\right)=2\left(g(3)-g(2)^{2}\right)\left(\begin{array}{l}
n \\
2
\end{array}\right), \\
& (n+1) ! A_{n+1, n-2}-n ! A_{n, n-3}-n ! g(2) n A_{n, n-2} \\
= & 15 g(2)^{3}\left(\begin{array}{l}
n \\
5
\end{array}\right)+20 g(2) g(3)\left(\begin{array}{l}
n \\
4
\end{array}\right)+6 g(4)\left(\begin{array}{l}
n \\
3
\end{array}\right) \\
& -g(2) n\left(3 g(2)^{2}\left(\begin{array}{l}
n \\
4
\end{array}\right)+2 g(3)\left(\begin{array}{l}
n \\
3
\end{array}\right)\right) \\
= & g(2)^{3}\left(15\left(\begin{array}{l}
n \\
5
\end{array}\right)-3 n\left(\begin{array}{l}
n \\
4
\end{array}\right)\right)+g(2) g(3)\left(20\left(\begin{array}{l}
n \\
4
\end{array}\right)-2 n\left(\begin{array}{l}
n \\
3
\end{array}\right)\right) \\
& +6 g(4)\left(\begin{array}{l}
n \\
3
\end{array}\right) \\
= & -12 g(2)^{3}\left(\begin{array}{l}
n \\
4
\end{array}\right)+g(2) g(3)(3 n-15)\left(\begin{array}{l}
n \\
3
\end{array}\right)+6 g(4)\left(\begin{array}{l}
n \\
3
\end{array}\right) .
\end{aligned}
$$

Going back we observe that the coefficient of $X^{n-1}$ of

$$
\begin{aligned}
& (n+1) ! P_{n+1}^{g}(X)-n ! X P_{n}^{g}(X)-n ! g(2) n P_{n}^{g}(X) \\
& -(n-1) ! 2\left(g(3)-g(2)^{2}\right)\left(\begin{array}{l}
n \\
2
\end{array}\right) P_{n-1}^{g}(X)
\end{aligned}
$$

equals 0 .

For the coefficient of $X^{n-2}$ we obtain now

$$
\begin{aligned}
& (n+1) ! A_{n+1, n-2}-n ! A_{n, n-3}-n ! g(2) n A_{n, n-2} \\
& -(n-1) ! 2\left(g(3)-g(2)^{2}\right)\left(\begin{array}{l}
n \\
2
\end{array}\right) A_{n-1, n-2} \\
= & -12 g(2)^{3}\left(\begin{array}{l}
n \\
4
\end{array}\right)+g(2) g(3)(3 n-15)\left(\begin{array}{l}
n \\
3
\end{array}\right)+6 g(4)\left(\begin{array}{l}
n \\
3
\end{array}\right) \\
& -2\left(g(3)-g(2)^{2}\right)\left(\begin{array}{l}
n \\
2
\end{array}\right) g(2)\left(\begin{array}{c}
n-1 \\
2
\end{array}\right) \\
= & g(2)^{3}\left(-12\left(\begin{array}{l}
n \\
4
\end{array}\right)+2\left(\begin{array}{l}
n \\
2
\end{array}\right)\left(\begin{array}{c}
n-1 \\
2
\end{array}\right)\right) \\
& +g(2) g(3)\left((3 n-15)\left(\begin{array}{c}
n \\
3
\end{array}\right)-2\left(\begin{array}{l}
n \\
2
\end{array}\right)\left(\begin{array}{c}
n-1 \\
2
\end{array}\right)\right)+6 g(4)\left(\begin{array}{c}
n \\
3
\end{array}\right) \\
= & 6\left(g(2)^{3}-2 g(2) g(3)+g(4)\right)\left(\begin{array}{l}
n \\
3
\end{array}\right) .
\end{aligned}
$$


This means the polynomial

$$
\begin{aligned}
& (n+1) ! P_{n+1}^{g}(X)-n ! X P_{n}^{g}(X)-n ! g(2) n P_{n}^{g}(X) \\
& -(n-1) ! 2\left(g(3)-g(2)^{2}\right)\left(\begin{array}{c}
n \\
2
\end{array}\right) P_{n-1}^{g}(X)
\end{aligned}
$$

can only be 0 if $g(2)^{3}-2 g(2) g(3)+g(4)=0$.

Example 3. For $g(n)=\sigma(n)$, we obtain $27-24+7=10 \neq 0$.

Proof of Lemma 3. From the recursive definition (1) we obtain

$$
\begin{aligned}
\sum_{m=0}^{n} A_{n, m} X^{m} & =P_{n}^{g}(X)=\frac{X}{n} \sum_{k=1}^{n} g(k) P_{n-k}^{g}(X) \\
& =\frac{1}{n} \sum_{k=1}^{n} g(k) \sum_{m=0}^{n-k} A_{n-k, m} X^{m+1} \\
& =\frac{1}{n} \sum_{m=1}^{n} \sum_{k=1}^{n-m+1} g(k) A_{n-k, m-1} X^{m}
\end{aligned}
$$

In particular $A_{n, n}=\frac{1}{n} A_{n-1, n-1}=\frac{1}{n !}$. (This follows by induction. In the cases of the other coefficients below also induction is applied.) Further,

$$
\begin{aligned}
A_{n, n-1} & =\frac{1}{n}\left(A_{n-1, n-2}+g(2) A_{n-2, n-2}\right)=\frac{1}{n}\left(A_{n-1, n-2}+\frac{g(2)}{(n-2) !}\right) \\
& =\frac{1}{n !}\left((n-1) ! A_{n-1, n-2}+g(2)(n-1)\right)=\frac{g(2)}{n !}\left(\begin{array}{l}
n \\
2
\end{array}\right), \\
A_{n, n-2} & =\frac{1}{n}\left(A_{n-1, n-3}+g(2) A_{n-2, n-3}+g(3) A_{n-3, n-3}\right) \\
& =\frac{1}{n}\left(A_{n-1, n-3}+\frac{g(2)^{2}}{(n-2) !}\left(\begin{array}{c}
n-2 \\
2
\end{array}\right)+\frac{g(3)}{(n-3) !}\right) \\
& =\frac{1}{n !}\left((n-1) ! A_{n-1, n-3}+3 g(2)^{2}\left(\begin{array}{c}
n-1 \\
3
\end{array}\right)+2 g(3)\left(\begin{array}{c}
n-1 \\
2
\end{array}\right)\right) \\
& =\frac{1}{n !}\left(3 g(2)^{2}\left(\begin{array}{l}
n \\
4
\end{array}\right)+2 g(3)\left(\begin{array}{l}
n \\
3
\end{array}\right)\right),
\end{aligned}
$$




$$
\begin{aligned}
A_{n, n-3}= & \frac{1}{n}\left(A_{n-1, n-4}+g(2) A_{n-2, n-4}+g(3) A_{n-3, n-4}+g(4) A_{n-4, n-4}\right) \\
= & \frac{1}{n}\left(A_{n-1, n-4}+\frac{g(2)}{(n-2) !}\left(3 g(2)^{2}\left(\begin{array}{c}
n-2 \\
4
\end{array}\right)+2 g(3)\left(\begin{array}{c}
n-2 \\
3
\end{array}\right)\right)\right. \\
& \left.+g(3) \frac{g(2)}{(n-3) !}\left(\begin{array}{c}
n-3 \\
2
\end{array}\right)+\frac{g(4)}{(n-4) !}\right) \\
= & \frac{1}{n !}\left((n-1) ! A_{n-1, n-4}+15 g(2)^{3}\left(\begin{array}{c}
n-1 \\
5
\end{array}\right)+8 g(2) g(3)\left(\begin{array}{c}
n-1 \\
4
\end{array}\right)\right. \\
& \left.+12 g(2) g(3)\left(\begin{array}{c}
n-1 \\
4
\end{array}\right)+6 g(4)\left(\begin{array}{c}
n-1 \\
3
\end{array}\right)\right) \\
= & \frac{1}{n !}\left(15 g(2)^{3}\left(\begin{array}{c}
n \\
6
\end{array}\right)+20 g(2) g(3)\left(\begin{array}{c}
n \\
5
\end{array}\right)+6 g(4)\left(\begin{array}{c}
n \\
4
\end{array}\right)\right) .
\end{aligned}
$$

\section{Open Question}

We proved that the polynomials $P_{n}^{g}(X)$ attached to $g(n)=n$ are irreducible and have real roots. Moreover, the roots interlace. Actually we were able to identify these polynomials with certain Laguerre polynomials which satisfy a three term recurrence relation. As indicated in the introduction, our main interest are the roots of $P_{n}^{\sigma}(X)$, since these roots dictate the vanishing properties of powers the Dedekind eta function [HNR17, HLN18], including the Lehmer conjecture [Le47]. Since $n \leq \sigma(n) \leq n^{2}$ properties of $P_{n}^{\sigma}(X)$ are expected to be deduced from the polynomials attached to $g(n)=n$ and $g(n)=n^{2}$ (as for example the size of the coefficients of $\left.P_{n}^{\sigma}(X)\right)$. We have for $g(n)=n^{2}$ :

$$
\widetilde{P}_{n}(X)=\sum_{k=0}^{n-1} A_{n, k} \frac{X^{k}}{(k+1) !}, \quad \text { where } A_{n, k}=\left(\begin{array}{c}
n+k \\
2 k+1
\end{array}\right) \in \mathbb{Z} .
$$

We have shown in this paper that these polynomials satisfy a four term recursion and that they are not orthogonal. Numerical calculations for $n \leq$ 100 indicate that the polynomials are also irreducible. For $n \leq 100$ we have checked that they have real roots and that the roots interlace. We end with this observation and ask the question of whether these facts hold for general $n$.

Acknowledgement. The paper was started when the two first named authors had been invited guests at the Max-Planck-Institute for Mathematics in Bonn in 2018. Both authors thank the MPIM for its hospitality and excellent research conditions. The first and third named authors thank the German University of Technology in Oman for supporting their research. Finally we would also like to thank the reviewer. 


\section{REFERENCES}

[Ai07] M. Aigner: Discrete Mathematics. American Mathematical Society (2007).

[AF03] M. Allen, M. Filaseta: A generalization of a second irreducibility theorem of I. Schur, Acta Arith., 109 (2003), 65-79.

[Do16] B. Doman: The Classical Orthogonal Polynomials. World Scientific (2016).

[HLN18] B. Heim, F. Luca, M. Neuhauser: On cyclotomic factors of polynomials related to modular forms. The Ramanujan Journal (2018) DOI: 10.1007/s11139-0179980-8.

[HN18] B. Heim, M. Neuhauser: Log-concavity of recursively defined polynomials. preprint, submitted.

[HNR17] B. Heim, M. Neuhauser, and F. Rupp: Fourier coefficients of powers of the Dedekind eta function. The Ramanujan Journal (2017) DOI: 10.1007/s11139017-9923-4.

[HNR18] B. Heim, M. Neuhauser, and F. Rupp: Imaginary powers of the Dedekind eta function. Experimental Mathematics (2018) DOI: 10.1080/10586458.2018.1468288.

[Le47] D. Lehmer: The vanishing of Ramanujan's $\tau(n)$. Duke Math. J. 14 (1947), 429-433.

[On03] K. Ono: The Web of Modularity: Arithmetic of the Coefficients of Modular Forms and q-series. Conference Board of Mathematical Sciences 102 (2003).

[Sc29] I. Schur: Einige Sätze über Primzahlen mit Anwendungen auf Irreduzibilitätsfragen, II, Sitzungsber. Preuss. Akad. Wiss. Berlin, Phys.-Math. Kl. (1929), 370-391.

[Sc31] I. Schur: Affektlose Gleichungen in der Theorie der Laguerreschen und Hermiteschen Polynome, J. Reine Angew. Math., 165 (1931), 52-58.

[WY05] Y. Wang, Y. N. Yeh: Polynomials with real zeros and Pólya frequency sequences, J. Combin. Theory Ser. A, 109 (2005), 63-74.

German University of Technology in Oman (GUtech), PO Box 1816, Athaibah PC 130, Sultanate of Oman

E-mail address: bernhard.heim@gutech.edu.om

School of Mathematics, University of the Witwatersrand, Private Bag 3, Wits 2050, Johannesburg, South Africa

Max-Planck-Institute for Mathematics, Vivatsgasse 7, 53111 Bonn, GerMANY

Department of Mathematics, Faculty of Sciences, University of Ostrava, 30 dubna 22, 70103 Ostrava 1, Czech Republic

E-mail address: Florian.Luca@wits.ac.za

German University of Technology in Oman (GUtech), PO Box 1816, Athaibah PC 130, Sultanate OF OMAN

E-mail address: markus.neuhauser@gutech.edu.om 